\title{
Content validation of a tool for assessing risks for drug-related problems to be used by practical nurses caring for home-dwelling clients aged $\geq 65$ years: a Delphi survey
}

\author{
Maarit S. Dimitrow • Sanna I. Mykkänen • \\ Saija N. S. Leikola • Sirkka-Liisa Kivelä • Alan Lyles • \\ Marja S. A. Airaksinen
}

Received: 31 January 2014 / Accepted: 14 May 2014

(C) Springer-Verlag Berlin Heidelberg 2014

\begin{abstract}
Purpose Home care services are becoming a critically important part of health care delivery as populations are aging. Those using home care services are increasingly older, more frail than previously, and use multiple medications, making them vulnerable to drug-related problems (DRPs). Practical nurses (PN) visit homedwelling aged clients frequently and, thus, are ideally situated to identify potential DRPs and, if needed, to communicate them to physicians for resolution. This study developed and validated the content of a tool to be used by PNs for assessing DRP risks for their homedwelling clients aged $\geq 65$ years.

Methods The first draft of the tool was based on two systematic literature reviews and clinical experience of our research group. Content validity of the tool was determined by a threeround Delphi survey with a panel of 18 experts in geriatric
\end{abstract}

Electronic supplementary material The online version of this article (doi:10.1007/s00228-014-1699-5) contains supplementary material, which is available to authorized users.

M. S. Dimitrow $(\bowtie) \cdot$ S. I. Mykkänen · S. N. S. Leikola •

S.-L. Kivelä • A. Lyles $\cdot$ M. S. A. Airaksinen

Clinical Pharmacy Group, Faculty of Pharmacy, University of

Helsinki, PL 56 (Viikinkaari 9C), 00014 Helsinki, Finland

e-mail: maarit.dimitrow@helsinki.fi

S. I. Mykkänen

e-mail: mykkanen.sanna@gmail.com

S. N. S. Leikola

e-mail: saija@leikola.fi

S.-L. Kivelä

e-mail: sirkiv@utu.fi

A. Lyles

e-mail: alanlyles@comcast.net

M. S. A. Airaksinen

e-mail: marjaairaksinen@gmail.com care and pharmacotherapy. An agreement by $\geq 80 \%$ of the panel on an item was required.

Results The final tool consists of 18 items that assess risks for DRPs in home-dwelling aged clients. It is divided into four sections: (1) Basic Client Data, (2) Potential Risks for DRPs in Medication Use, (3) Characteristics of the Client's Care and Adherence, and (4) Recommendations for Actions to Resolve DRPs.

Conclusions The Delphi process resulted in a structured DRP Risk Assessment Tool that is focused on the highest priority DRPs that should be identified and resolved. The tool also assists the PNs to identify solutions to these problems, which is a unique feature compared to similarly purposed prior tools.

Keywords Geriatric pharmacotherapy · Adverse drug reactions - Drug-related problems - Risk management . Patient safety - Primary care

S.-L. Kivelä

Department of Family Medicine, Institute of Clinical Medicine, University of Turku, Turku, Finland

S.-L. Kivelä

Unit of Family Medicine, Turku University Hospital, Turku, Finland

S.-L. Kivelä

Satakunta Hospital District, Pori, Finland

A. Lyles

School of Health and Human Services, University of Baltimore, 1420 N Charles St., Baltimore, MD 21201, USA 


\section{Introduction}

A drug-related problem (DRP) is an event or circumstance involving drug therapy that actually or potentially interferes with desired health outcomes [1]. Age-related anatomical and physiological alterations combined with the presence of comorbidity and polypharmacy make pharmacotherapy of the aged both challenging and vulnerable to DRPs [2, 3]. These risks can be related to the pharmacological characteristics of medicines or to medicine use processes of individual patients $[4,5]$.

Long-term home care services for the aged are becoming a critically important part of public health care delivery in Finland as it is in many Western countries [6, 7]. An increasing proportion of older people, cost increases, and consumer preferences are among the major influences on this shift. Aged people in home care are increasingly older and more frail than previously and use multiple medications $[8,9]$. They see physicians infrequently but may have more regular, even daily, encounters with home care practical nurses (PNs) [10]. Thus, PNs are in a key position for identifying clients at risk for adverse drug reactions (ADRs) and other DRPs. However, PNs need tools that are feasible for use in routine practice and that focus on identifying the most critical pharmacotherapy risks of the aged.

Even though numerous explicit and implicit criteria for potentially inappropriate medications for the aged have been established [11, 12], there is a lack of validated tools designed to be used by PNs in home care that would screen aged clients at high risk for DRPs and communicate the results to the entire care team. The existing tools [13-21] are meant to be completed by pharmacists [13], by other health and social care workers [15], or by the patients themselves during clinic visits $[14,17,19,21]$. Moreover, some of the tools are nonspecific for age or geriatric application [13, 17, 18, 21]. We are aware of two nurse-administered risk assessment tools designed for the aged $[16,20]$. They primarily focus on nonadherence [16] or nonadherence and the complexity of the patient's medication regimen [20] but ignore geriatric-specific ADRs or medicines that pose greater risks for the aged. In addition, these tools were not designed to be used by PNs, who have a 3-year vocational qualification with modest training in pharmacotherapy but who are the ones who most commonly make home visits and work most closely with the home-dwelling aged clients [22].

The present study developed and content validated a DRP Risk Assessment Tool for use by PNs taking care of home-dwelling clients $\geq 65$ years. The tool is designed (1) to identify those aged $\geq 65$ years at high risk for adverse drug reactions or other DRPs and (2) to assist in recommending the potential risk clients intervening actions for optimizing their pharmacotherapy. The tool is intended to be used by PNs in the context of their routine home visits and to pose minimal administrative and time burdens for learning its routine use.

\section{Methods}

The DRP Risk Assessment Tool was developed in two major phases, namely by (1) developing a draft tool on the basis of two systematic literature reviews [11, the other one is unpublished (in preparation)] and the clinical expertise of the research group and (2) determining the content validity of the tool by a three-round Delphi survey [23] involving a panel of 18 experts in geriatric care and pharmacotherapy (Fig. 1).

\section{Systematic literature reviews}

The literature on existing criteria for assessing inappropriate prescribing in patients aged $\geq 65$ years was systematically reviewed according to the PRISMA statement criteria [11, 24]. In addition to the abovementioned systematic literature review on potentially inappropriate medications for the aged, we also systematically searched existing tools designed to screen aged patients at risk for DRPs. The following search terms were used: (elderly or aged or ageing), (medication-related problem or drug-related problem or drug-therapy problem), (risk), and (screening tool or tool or form). English language articles published during 1985-2010 were searched in the following databases: Evidence-Based Medicine database, Web of Knowledge, Scopus, and CINAHL.

\section{Development of the draft DRP Risk Assessment Tool}

According to the findings of the systematic review [11], we identified (1) medicines (e.g., theophylline, amiodarone, digoxin, fluoxetine) and medicine classes (e.g., anticholinergics, sedatives, neuroleptics, nonsteroidal anti-inflammatory drugs, diuretics, hypoglycemics) recognized to pose higher risks for the elderly and (2) potential geriatric-specific ADRs caused by these medicines. Second, according to the findings of the unpublished systematic review that focused on existing agespecific ( $\geq 65$ years) screening tools $(n=5)[14-16,19,20]$, we listed the most commonly mentioned prognostic indicators for DRPs. The review also revealed some other factors to be considered, particularly the structure of the tool and focusing the screening on potentially harmful ADRs instead of producing a long list of problematic medicines [19]. Both of these systematic literature reviews' findings were integrated into the draft tool. Our research group's clinical experience was used in this phase, as well as that of an experienced geriatrician who was not a member of the research group.

As PNs' training concentrates mainly on supportive and technical aspects of nursing rather than on pharmacotherapy 


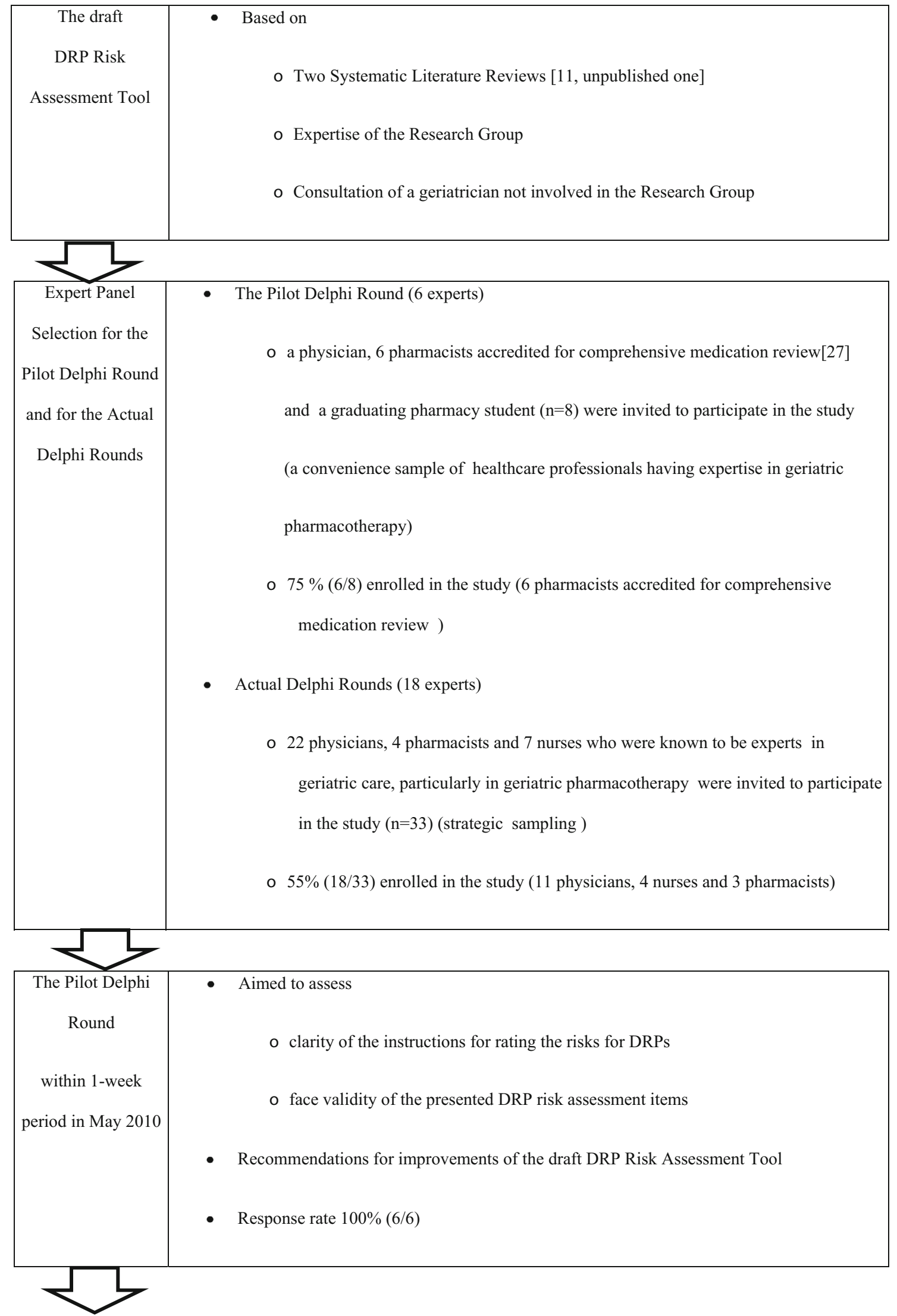

Fig. 1 Outline of the development of the Drug-Related Problem (DRP) Risk Assessment Tool and validation of its content by Delphi method. ${ }^{a}$ each component of multi-component items are counted as single items. ADR = Adverse-Drug Reaction, DRP = Drug-Related Problem [1] 


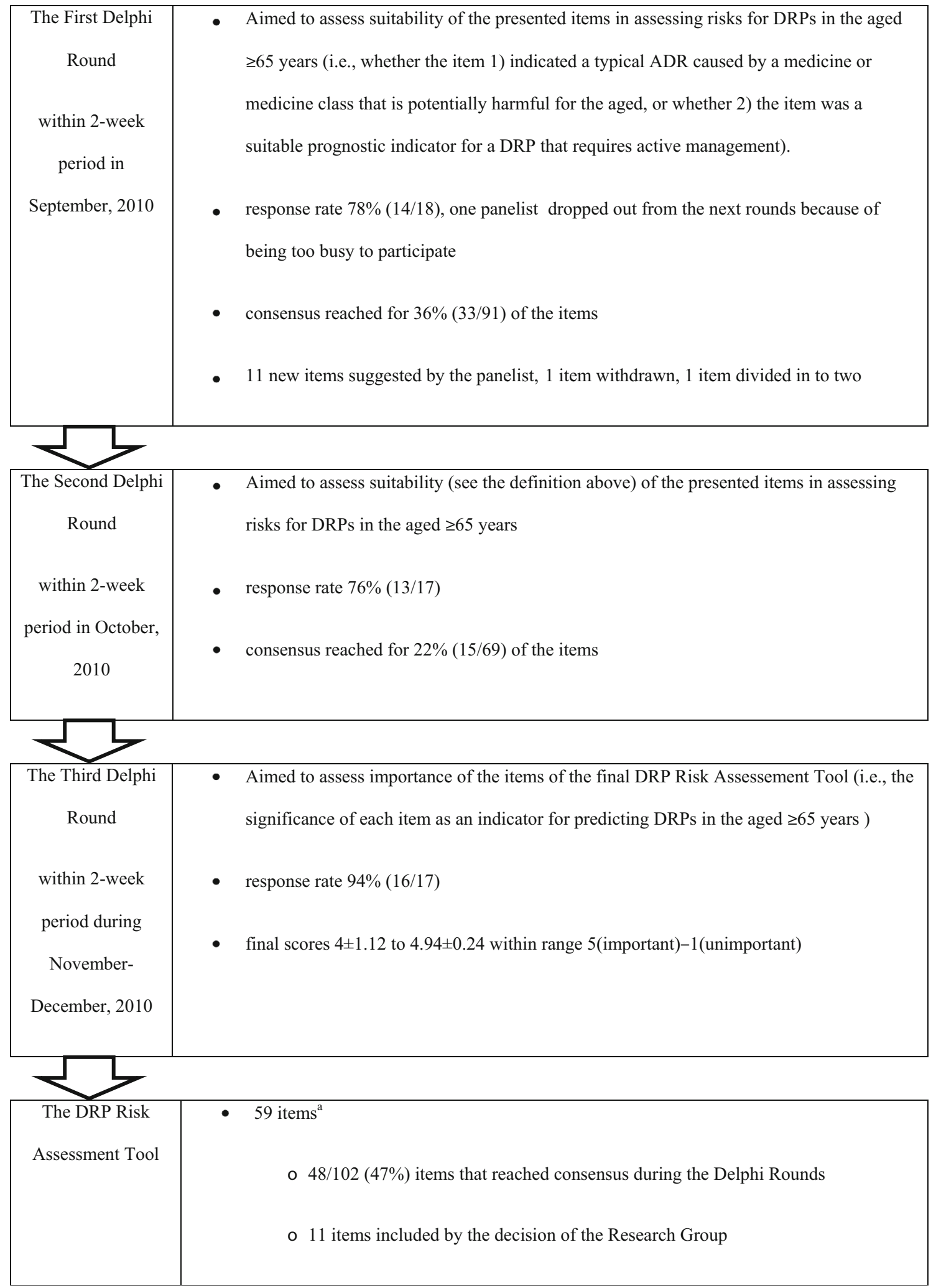

Fig. 1 (continued)

[22], lay language terms were used throughout the draft tool to make it easy to use for them. For example, instead of asking directly whether the client uses statins, the following wording was used: "Does the client use medicines that are intended to lower the cholesterol level (statins)?" For the sake of clarity, International Nonproprietary Names (INNs) of medicines 
were avoided whenever possible. Instead, we asked if the client suffered from symptoms that would typically suggest ADRs related to medicines and medicine classes that are problematic for the aged.

\section{The Delphi method}

The Delphi method is a systematic technique for formulating a group judgment concerning subject matter for which the information is incomplete or lacking entirely $[23,25]$. Its validity relies on a panel of experts having been carefully selected, that their individual judgments be provided independently and anonymously via questionnaires in two or more rounds, and that there be aggregated feedback between rounds. In this study, three Delphi rounds were performed online via the software called eDelfoi [26].

The process for identifying and selecting the expert panel (Fig. 1) resulted in a final panel comprising 11 physicians, 4 nurses, and 3 pharmacists. The primary criterion for selecting each panelist was that they have extensive clinical experience in geriatric care, including pharmacotherapy. The physicians' $(n=11)$ expertise spanned clinical pharmacology, general practice, geriatrics, internal medicine, and nephrology. Five of them had higher academic degrees (PhDs), and two were specializing in neurology and general practice. One of the nurses and two of the pharmacists had a $\mathrm{PhD}$ degree.

The Delphi rounds for content validation of the DRP Risk Assessment Tool

We pilot tested the draft tool by conducting a pilot Delphi round before the actual rounds (Fig. 1). A few minor modifications were then made according to the panelists' proposals.

Two authors independently analyzed each Delphi round's responses (including the pilot round), and their results were compared. The few emergent disagreements were resolved between the rounds by discussing them in the research group meetings, with the final form of the next round's tool being decided in those meetings.

Before each Delphi round, it was stressed to the panelists that the final Tool was meant to be administered by PNs and that the panelists should consider a typical PN's ability to answer the items. The panelists' guidance also emphasized the goal of developing a tool which (1) focuses on the most important predictors for DRPs in the aged, (2) is easy to use in the time constraints of routine practice, and (3) requires only modest training for PNs.

During the first and second Delphi rounds, the panelists were asked to rate the suitability of every item in the evolving versions of the draft tool (including each individual component of multicomponent items) in assessing DRP risks for clients $\geq 65$ years. Depending on the item, they were asked to rate whether the item (1) indicated a typical ADR caused by a medicine or medicine class that is potentially harmful for the aged or whether (2) the item was a suitable prognostic indicator for a DRP that requires active management. Round one also included a final open-ended question: If you think that the draft risk assessment tool ignores some essential aspects of medication risks in routine community clinical practice, please identify each of them.

The second round survey included items for which consensus had not yet been reached; they were then modified according to the panelists' recommendations. New items suggested by the panelists were added. The panelists also received a list of items on which consensus had been reached in the first round. After the second round the research group decided to add 11 items for which consensus had not been reached during the two first rounds (Table 1). The expert panelists were informed of this decision at the beginning of the third round and invited to the items individually.

During the third final round, panelists rated the importance of each of the final DRP Risk Assessment Tool items (i.e., the significance of each item as an indicator for predicting DRPs). Multicomponent items were rated as one item (Table 1, main items 5, 6, 8, and 14), with importance being rated with the alternatives "important," "moderately important," "cannot say," "moderately unimportant," or "unimportant". In addition to rating the importance of individual items, panelists were asked to identify symptoms listed in the final tool that would be suggestive of ADRs requiring immediate actions (i.e., contacting the physician) (Table 1, item 8).

\section{Data analysis}

Items that $\geq 80 \%$ of the panelists rated as suitable were included in the final DRP Risk Assessment Tool. A similar consensus criterion was applied to the panelists' ratings of the symptoms requiring immediate action. The importance of each main item in the final tool was scored from 5 to $1(5=$ important, $1=$ unimportant). The mean ratings and standard deviations (SD) are reported.

\section{Results}

Panelists achieved consensus on 48 of 102 items ( $47 \%$ ) (each main item and each individual item of the final tool's multicomponent items are discussed here as items; see Table 1). In addition to these 48 consensus items, 11 items were retained according to the research group's judgment (Fig. 1), resulting in the 59-item final DRP Risk Assessment Tool. This final version of the tool consists of four main sections: (1) Basic Client Data, (2) Potential Risks for DRPs in Medication Use, (3) Characteristics of the Client's Care and Adherence, and (4) Recommendations for Actions to Resolve DRPs (Table 1). 
Table 1 The Drug-Related Problem (DRP) Risk Assessment Tool

$\begin{array}{ll}\text { Basic Client Data } & \\ \text { Name } & \\ \text { Identity number } & \\ \text { Age } & \text { Year } \\ \text { Gender } & \\ \quad \text { Male } & \\ \quad \text { Female } & \square\end{array}$

Does the client live alone?

Does the client have an up-to-date medication card/list?

$\begin{array}{ll}\text { Yes } & \text { No } \\ \square & \square \\ \text { Yes } & \text { No }\end{array}$

No

No

$\square$

Who administers the client's medicines?

\section{Potential Risks for DRPs in Medication Use}

1. Does the client have seven $^{\mathrm{a}}$ or more prescription medicines in current $\quad$ Yes No regular use? (excluding basic creams)

2. Does the client take 12 or more medicine doses regularly each day (excluding basic creams)?

$\square \quad$ प

Yes $\quad$ No

口

Example of counting the doses: Drug 1: 1 tablet 3 times a day=3 doses, Drug 2: 1 dose 2 times a day=2 doses, i.e., in total 5 doses a day

3. Is the client currently taking medicines for three or more diseases or Yes No symptoms? (including acute diseases)

4. Has the client started a new medicine in the last 4 weeks? (excluding Yes No different brands of the same active ingredient) $\quad \square \quad \square$

5. Does the client use medicines that... Yes No

a. relieve pain by reducing inflammation (does not apply to paracetamol)? $\quad \square \quad \square$

b. elevate the rate of urination (diuretics) ${ }^{\mathrm{a}} \quad \square \quad \square$

c. are intended to lower the cholesterol level (statins) ${ }^{\mathrm{a}} \quad \square \quad \square$

d. the physician does not know about?

6. Does the client use any of the following medicines (please check the ones used)? (the list contains medicines with a narrow therapeutic index, medicines for which regular monitoring would be necessary and medicines that otherwise are problematic for the aged)

$\begin{array}{llll}\text { Amiodarone }^{\mathrm{a}} & \square & \text { Lithium }^{\mathrm{a}} & \square \\ \text { Carbamazepine } & \square & \text { Methotrexate }^{\mathrm{a}} & \square \\ \text { Digoxin } & \square & \text { Theophylline }^{\mathrm{a}} & \square \\ \text { Fluoxetine } & \square & \text { Warfarin } & \square\end{array}$

7. Has the client used over-the-counter (OTC) medicines or vitamin, mineral Yes No or herbal products in the past 2 weeks? State which ones.

8. Has the client had any of the following symptoms in the last 4 weeks? (Please tick below "yes" if it has been ongoing and add another tick in the right column, if the symptom is a new one $=$ a symptom that had first occurred within the last 4 weeks)

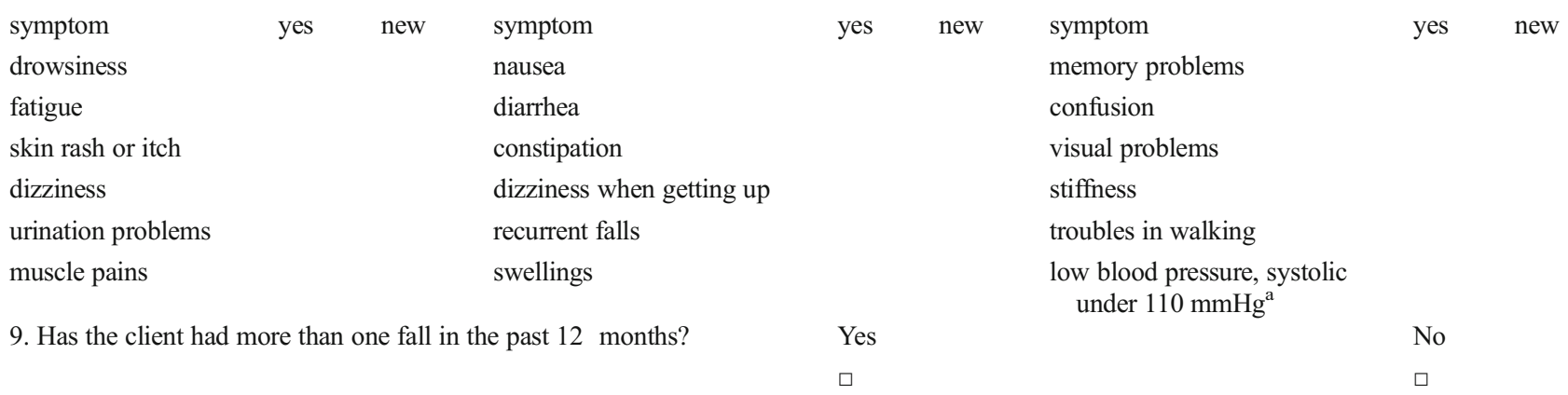


Table 1 (continued)

10. Has the client/relative/visitor noticed any changes in client's condition Yes No that could indicate adverse drug reactions related to changes in medicines $\square$ regimen?

Characteristics of the Client's Care and Adherence

a) Health, health care setting and care taking physician

11. Does the client have three or more chronic diseases? Yes

12. Has the client been in short term care (e.g., interval care) in hospital, Yes nursing home, sheltered housing, health centre ward or some other $\quad \square$ institution in the past 4 weeks?

13. Does the client have more than one physician involved in his/her care? Yes (e.g., general practitioners, specialists, private practitioners)

b) Adherence

14. Has the client had troubles in $\quad$ Yes

a. remembering to take the medicines?

b. following the medicines regimen?

c. knowing what his or her medicines are used for?

d. affording the medicines (i.e., economic problems)?

e. opening the drug bottles or packages or managing with medicines related therapeutic devices?

15. Does the client consciously sometimes take medicines differently than Yes prescribed?

Yes

No

Yes

No

口

Yes No

$\square \quad \square$

$\square \quad \square$

$\square$

$\square \quad \square$

口

$\square \quad \square$

Yes $\quad$ No

16. Is the client (or his/her caregiver) aware of the client's diseases and their Yes treatments?

17. Is the client (or his/her caregiver administering the medication) aware of Yes the medicines that the client uses?

$\square$

No

18. Have the client's relatives/proxies expressed their concern about the Yes

client's medicine use?

Yes $\quad$ No

Recommendations for Actions to Resolve DRPs (several items can be selected if necessary):

a) Comprehensive Medication Review

Yes No

b) Using dose dispensing device

c) Automated dose dispensing ${ }^{\mathrm{a}}$

d) Visiting the personal physician

e) Visiting a diabetes or asthma nurse in the health centre or the diabetes or

$\square \quad \square$

asthma pharmacist in the pharmacy ${ }^{\mathrm{a}}$

f) Weekly control visits by a home care nurse

g) Follow-up of the client's condition (repeating the risk test) ${ }^{\mathrm{a}}$

Client's permission for possible intervening actions?

$\begin{array}{ll}\square & \square \\ \square & \square \\ \square & \square \\ \square & \square \\ & \square \\ \square & \square \\ \square & \square \\ \square & \square\end{array}$

${ }^{a}$ Retained in the DRP Risk Assessment Tool by judgment of the research group

The contents of these four sections are described more in detail in the following paragraphs.

\section{Section 1: Basic Client Data}

The first section of the DRP Risk Assessment Tool focuses on basic client demographic data, such as age and gender (Table 1). The Delphi survey rounds yielded the following two additions to the basic client information: (1) an indicator of whether the client has an up-to-date medication card/list and (2) who administers the client's medications. These items are relevant for identifying whether the client's medication management process is fragmented, thus posing a risk for unrecognized and unsolved DRPs.

\section{Section 2: Potential Risks for DRPs in Medication Use}

Section 2 consists of ten main items, of which three are multicomponents, representing 38 single items in total. This section of the tool focuses on symptoms potentially suggestive of ADRs (3 main items with 21 single items) or medicines potentially harmful or problematic for clients aged $\geq 65$ years ( 2 main items with 12 single items, Table 1 ). The list contains medicines having a narrow therapeutic index, medicines for which regular monitoring would be necessary, or medicines that are otherwise problematic for the aged. The section also includes items indicating risks posed by polypharmacy (three items), initiation of a new medication (one item), and concomitant use of nonprescription medicines or vitamins with prescription medicines (one item). 
Section 2's items are mostly binary, with "yes" or "no" options for indicating the client's condition. Of the 18 potential ADRs listed in item 8 (Table 1), three were rated by the expert panel as symptoms requiring immediate actions (fatigue, recurrent falls, and confusion). However, we decided not to emphasize these three symptoms in the final DRP Risk Assessment Tool because that could lead to underestimating the potential significance of other symptoms. Instead, we included an additional rating option for whether the symptom is new (see Table 1, item 8). This addition was suggested by the panelists in the first Delphi round. The research group qualified it as meaning a symptom that had first occurred within the last 4 weeks. In the second Delphi round, the panelists reached consensus for this addition to rating the symptoms.

Section 3: Characteristics of the Client's Care and Adherence

Section 3 consists of eight main items with one multicomponent item, representing 12 single items in total. It includes information about the client's health, adherence to their therapeutic regimen and involvement in one's care, the health care setting, health care units recently visited by the client, and the number of care-taking physicians (Table 1). All of the section's items have a "yes" or "no" rating option.

\section{Section 4: Recommendations for Actions to Resolve DRPs}

This section encompasses seven interventions that PNs can recommend for resolving potential DRPs for those at risk (Table 1). The recommendations that PNs make need to be based on a risk assessment resulting from the information gathered by using the DRP Risk Assessment Tool.

Ranking the importance of items included in the final DRP Risk Assessment Tool

Table 2 contains the rating results of the Delphi panel's third round of the importance of the items included in the final Drug-Related Problem (DRP) Risk Assessment Tool (Online Resource 1).

The panelists ranked two items as the most important for predicting DRP risks in the aged $\geq 65$ years: (1) the item indicating whether the client has an up-to-date medication list and (2) the item assessing the client's adherence to his/her medication.

All of the items in the final tool were rated either as important or moderately important in predicting DRP risks for those $\geq 65$ years. Mean scores varied from $4.94 \pm 0.24$ to $4 \pm$ 1.12, within the range of 5 (important)-1 (unimportant). More dispersion was observed in items with the lowest mean scores. The items with the highest mean scores also had the smallest standard deviations, indicating high concordance between the panelists.

\section{Discussion}

\section{DRP Risk Assessment Tool for PNs}

Developing the DRP Risk Assessment Tool followed a rigorously selective process to produce an instrument that could be used by PNs supporting medication management for homedwelling clients $\geq 65$ years but who have only modest formal training in pharmacotherapy. A three-round Delphi process yielded consensus ( $\geq 80 \%$ agreement on an item) on 48 DRP risk assessment items and a final agreement on the resulting 59-item tool.

In addition to indicating potential risks from the pharmacological effects of the medications, particularly ADRs, the DRP Risk Assessment Tool includes measures on risks related to poor adherence and to poor medication management, such as not involving clients and/or their caregivers in care, infrequent follow-ups, and poor coordination of care among the client's health care providers. The importance of adherence and medication management for minimizing DRP risks was confirmed by the Delphi panel experts who gave their final highest importance ratings to items indicating whether the client has a timely medication card/list and whether the client adheres to his/her medication. These indicators, either one or both, are missing or have had a minor role in previous tools that assess DRP risks in the aged [14-16, 19, 20] or criteria for inappropriate prescribing for the aged $[11,12]$.

Another feature of the final DRP Risk Assessment Tool is that, in addition to assisting PNs in identifying home-dwelling aged clients who have or who are at risk for DRPs that require actions, the tool actually guides the selection of such actions. Most of them are related to consulting a physician, another health care provider, or a specially trained pharmacist to review the client's medications and condition [4, 27]. Some clients may also benefit from automated dose dispensing for their medications (i.e., medicines are packed in unit-dose bags according to administration times), a service available to primary care patients throughout Finland [5, 28]. The tool also increases awareness among PNs and the aged of options to improve medication safety (e.g., comprehensive medication reviews, dose dispensing, and consulting an accredited pharmacist).

One third of the individual items in the DRP Risk Assessment Tool are indicators of potentially harmful ADRs. The number of these is high, even though over half of the initially nominated ADRs (28/46, $61 \%$ ) and most of the initially suggested problematic medicines (14/18, 78\%) were excluded during the successive Delphi rounds. This indicates the importance of taking ADRs into account as an integral part of medication use and of identifying ways to identify them early and manage them. The DRP Risk Assessment Tool explicitly guides PNs in the detection of 
Table 2 Rating results of Delphi panel's third round: the importance of the items included in the final drug-related problem (DRP) Risk Assessment $\mathrm{Tool}^{\mathrm{a}}$ (i.e., the significance of each item as an indicator for predicting DRPs in the aged $\geq 65$ years)

Items of the DRP Risk Assessment Tool in order of the mean scores
Number of panelists who rated the importance of Mean the items as score \pm SD

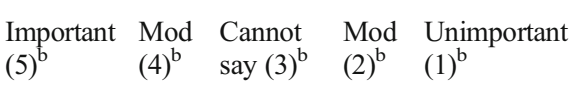

15

$4.94 \pm 0.24$

142

$4.88 \pm 0.33$
1. Does the client have an up-to-date medication card/list?

2. Has the client had troubles in

a. Remembering to take the medicines?

b. Following the medicines regimen?

c. Knowing what his or her medicines are used for?

d. Affording the medicines (i.e., economic problems)?

e. Opening the drug bottles or packages or managing with medicines related therapeutic devices?

3. Who administers the client's medicines?

4. Is the client (or his/her caregiver administering the medication) aware of the medicines that the client uses?

5. Has the client had any of the following symptoms in the last 4 weeks? (Please tick below "yes" if it has been ongoing and add another tick in the right column, if the symptom is a new one $=$ a symptom that had first occurred within last 4 weeks): drowsiness, fatique, skin rash or itch, dizziness, urination problems, muscle pains, nausea, diarrhea, constipation, dizziness when getting up, recurrent falls, swellings, memory problems, confusion, visual problems, stiffness, troubles in walking, low blood pressure, systolic under $110 \mathrm{mmHg}$

6. Does the client have more than one physician involved in his/her care? (e.g., general practitioners, specialists, private practitioners)

7. Does the client have seven or more prescription medicines in current regular use? (excluding basic creams)

8. Is the client (or his/her caregiver) aware of the client's diseases and their treatments?

9. Has the client started a new medicine in the last 4 weeks? (excluding different brands of the same active ingredient $)^{\mathrm{c}}$

10. Have the client's relatives/proxies expressed their concern about the client's medicine use?

11. Has the client/relative/visitor noticed any changes in client's condition that could indicate adverse drug reactions related to changes in medicines regimen?

12. Does the client consciously sometimes take medicines differently than prescribed?

13. Has the client had more than one fall in the past 12 months? ${ }^{c}$

14. Does the client use any of the following medicines (please check the ones used)? (the 13 list contains medicines with a narrow therapeutic index, medicines for which regular monitoring would be necessary and medicines that otherwise are problematic for the aged): amiodarone, carbamazepine, digoxin, fluoxetine, lithium, methotrexate, theophylline, warfarin

15. Is the client currently taking medicines for three or more diseases or symptoms? (including acute diseases)

16. Does the client use medicines that

a. Relieve pain by reducing inflammation (does not apply to paracetamol)?

b. Elevate the rate of urination (diuretics)?

c. Are intended to lower the cholesterol level (statins)?

d. The physician does not know about?

17. Has the client been in short term care (e.g., interval care) in hospital, nursing home, 6 sheltered housing, health centre ward or some other institution in the past four weeks?

18. Has the client used over-the-counter (OTC) medicines or vitamin, mineral or herbal 6 products in the past 2 weeks? State which ones.

19. Does the client have three or more chronic diseases?

20. Does the client take 12 or more medicine doses regularly each day (excluding basic 7 creams)? Example of counting the doses: Drug 1: 1 tablet 3 times a day $=3$ doses, Drug 2: 1 dose 2 times a day $=2$ doses, i.e., in total 5 doses a day
$4.81 \pm 0.39$

$4.81 \pm 0.39$

$4.75 \pm 0.43$

\section{2}

11

13

10

\section{0}

11

10

13

$\begin{array}{ll}5 & 1 \\ 4 & \end{array}$

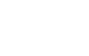

\footnotetext{
${ }^{a}$ The respondents were not asked to rate the importance of the item "The Recommendations for Actions to Resolve DRPs"

${ }^{\mathrm{b}}$ Scoring, "important" $=5$, "moderately important" $=4$, "cannot say" $=3$, "moderately unimportant" $=2$, "unimportant" $=1$

${ }^{\mathrm{c}} 15$ of 16 panelists rated of the importance of items 9 and 13
} 
specific symptoms associated with potential ADRs requiring attention. Otherwise, ADRs tend to be underreported among the aged as they may consider the symptoms to be a part of normal aging [29].

Expert panelists reached consensus on 48 of 102 initial items (47\%), which is less than the harvest in several other conceptually similar Delphi surveys reported in the health services' research literature [30-32]. The criterion for consensus $(80 \%)$ to determine attainment of this goal was high: Consequently, achievement of consensus was low. Therefore, we believe that only the most important items for identifying DRP risks in home-dwelling clients $\geq 65$ years were retained in the final DRP Risk Assessment Tool. Furthermore, our Delphi panel comprised physicians, pharmacists, and nurses who are leading experts in geriatric care and pharmacotherapy in Finland. Thus, they should have had the most comprehensive knowledge of typical potentially harmful ADRs and other risks for DRPs which require active management. In order to keep the panelists focused on the DRP Risk Assessment Tool's intended use, it was stressed to them before each Delphi round that the tool was meant to be administered by PNs and they have to consider a PN's ability to evaluate DRP risks for their individual home-dwelling aged clients.

One of the Delphi technique's limitations is that important items may be excluded if the panelists cannot reach consensus [33]. To avoid such omissions, we retained 11 items (Table 1) in the final DRP Risk Assessment Tool despite their lacking consensus. Based on scientific evidence and our research group's clinical expertise, those items were considered too important for the intended purpose to be excluded. Also, the Delphi panelists rated these retained items as important or moderately important during the third round survey, indicating their endorsement for including these additional 11 items in the final DRP Risk Assessment Tool.

The list of problematic medicines in the final DRP Risk Assessment Tool does not include hypoglycemics, which in several other reported criteria are identified as problematic for the aged [12, 34, 35]. Hypoglycemics are well documented in the literature as a leading cause of emergency department visits and hospitalizations in the aged [36]. The rationales for their omission are the following: (1) the most problematic oral hypoglycemics (chlorpropamide and glyburide) are no longer available in Finland and (2) the DRP Risk Assessment Tool includes items that would identify the symptoms of potential hypoglycemia.

Potential implications for practice

The DRP Risk Assessment Tool developed in this study is designed for direct use in clinical practice, and it has multiple potential applications for timely interventions that would improve the quality and safety of medicine use in home-dwelling clients $\geq 65$ years. As the PNs who visit home-dwelling aged clients would make a more accurate and standardized DRP risk assessment when guided by the tool, sharing their documented information with the client's physician and other health care providers involved in client's care can be readily integrated into routine practice. This may support physicians in making timely clinical interventions to manage a client's potential or manifest DRPs, e.g., harmful ADRs. The tool provides additional information for pharmacists who conduct comprehensive medication reviews [27]. Alternatively, the tool can assist in identifying clients in need of such reviews.

The DRP Risk Assessment Tool has educational implications. The routine use of the tool educates PNs and other health care providers involved in geriatric patients' care teams about geriatric pharmacotherapy and its role in clinical practice in home care. The tool also supports training on how to manage DRPs and to perform quality assurance in the medication use process, e.g., updating and harmonizing medication cards/lists.

Achieving optimal results from the DRP Risk Assessment Tool requires user education, periodic updates, and widespread adoption. If used consistently throughout a nation, the structured DRP Risk Assessment Tool will provide more timely and standardized information, harmonizing the way the health care personnel assess and communicate with each other to prevent, detect, and resolve DRPs of the clients aged $\geq 65$ years.

\section{Strengths and weaknesses of the study}

The Delphi technique is an accepted and commonly used method in health services research $[11,23]$, providing information in a valid and accessible manner. A Delphi survey's success, however, depends on the selection criteria, process, and quality of the expert panel $[25,37]$. To ensure a broad representation of skills and competence in geriatric care and pharmacotherapy, we invited panelists from three relevant but different expert professional groups: physicians, nurses, and pharmacists. Conducting the survey online facilitated broad geographic representation of that expertise. As the panelists were carefully selected among the best experts in geriatric care and pharmacotherapy in Finland, we believe they had a comprehensive understanding for validating the content of the DRP Risk Assessment Tool.

A multiprofessional 13-16-member expert panel responded in the three Delphi rounds (Fig. 1). This is consistent with or slightly greater than the panel size in several other Delphi surveys [11, 33, 34, 38, 39]. Thus, we believe that the number of participants is high enough to provide confidence in the stability of the results. Response rates in the Delphi rounds in the current study varied from 76 to $94 \%$, indicating 
the panelists' involvement and commitment to the development of the DRP Risk Assessment Tool.

Even though it was initially based on two systematic literature reviews [11, the unpublished one], the draft DRP Risk Assessment Tool may have missed some relevant aspects of patient care. That is why the expert panelists were asked to suggest additional items based on their clinical expertise. We also drew on the research group's special expertise in geriatric pharmacotherapy and geriatrics to complement the evidencebased origins for items in the final DRP Risk Assessment Tool. Thus, we believe that the development and validation processes have been rigorous enough to overcome potential deficiencies with the methodology.

It may be considered a limitation that our systematic literature review of potentially inappropriate medicines for the aged includes published papers until July 2010 [11]. However, we updated the literature review in 2012, including published papers until June 2012 [40]. Thus, our literature covers the main problematic medicines and medicine classes and is still up-to-date, including, e.g., the most recent update of the Beers Criteria in 2012 [12].

The DRP Risk Assessment Tool's content was validated in this study. The next step is to validate it in the field. We have already conducted a feasibility study among PNs in home care to test their ability to answer the items in the tool reliably and to assess the time spent completing the tool. Preliminary study results indicate that the tool is appropriate to a PN's skill level. The results of these analyses will be reported in detail in subsequent publications.

\section{Conclusion}

We have developed and content validated a DRP Risk Assessment Tool intended to be used by PNs in home care for home-dwelling clients $\geq 65$ years. The tool is designed for unintrusive, direct application in clinical practice. The Delphi process used to construct the tool resulted in a structured DRP Risk Assessment Tool that focuses on the identification and resolution of the highest priority DRP risks. In addition to identifying pharmacotherapeutic risks, the tool assists in finding solutions to these problems - a unique feature when the tool is compared to previous risk assessment tools.

\footnotetext{
Acknowledgments The following persons are acknowledged in the manuscript: Prof Kaisu Pitkälä (Unit of General Practice, Helsinki University Central Hospital, Helsinki, Finland, Department of General Practice and Primary Health Care, Faculty of Medicine, University of Helsinki, Helsinki, Finland) for consulting the preliminary questionnaire and Hannu Linturi, MSc, (Otava Folk High School in partnership with The Finnish Society for Future Studies and Finland Research Centre (University of Turku)) and Dr Anita Rubin (Finland Future Research Centre (University of Turku), The Finnish Society for Future Studies) for their valuable assistance during the Delphi process. We are also grateful to
}

Richard Stevenson, MSc, for linguistic help with the DRP Risk Assessment Tool, Thomas R Fulda, MA, for linguistic help with the manuscript, and Juhani Pohjola, $\mathrm{PhD}$, for assessing the legibility of the manuscript. The corresponding author (MD) affirms that she has listed everyone who contributed significantly to the work and has obtained written consent from all contributors who are not authors and are named in the Acknowledgments section.

Conflict of interest The authors declare that they have no conflicts of interest. No sources of funding were used to assist in the preparation of this study. Maarit Dimitrow received a grant for her $\mathrm{PhD}$ studies from the Finnish Cultural Foundation in 2012.

Author Contribution Study concept and design (MD,SL, SLK,AL,MA), Acquisition of data (MD,SM,S-LK,MA), Analysis and interpretation of data (MD,SM,SL,S-LK,AL,MA), Drafting the manuscript and the final approval of the version to be published (MD,SM,SL,S-LK,AL,MA).

\section{References}

1. Pharmaceutical Care Network Europe (PCNE) (2009) http://www.pcne. org/sig/drp/drug-related-problems.php Accessed 18 December 2013

2. ElDesoky ES (2007) Pharmacokinetic-pharmacodynamic crisis in the elderly. Am J Ther 14:488-498

3. Klotz U (2009) Pharmacokinetics and drug metabolism in the elderly. Drug Metab Rev 41:67-76

4. Leikola SN, Virolainen J, Tuomainen L, Tuominen RK (2003) Airaksinen MS (2012) Comprehensive medication reviews for elderly patients: findings and recommendations to physicians. J Am Pharm Assoc 52:630-633

5. Sinnemäki J, Saastamoinen LK, Hannula S, Peura S, Airaksinen M (2013) Starting an automated dose dispensing service provided by community pharmacies in Finland. Int J Clin Pharm. doi:10.1007/ s11096-013-9899-0

6. Teperi J, Porter ME, Vuorenkoski L, Baron JF (2009) The Finnish health care system: a value-based perspective, Sitra Reports 82 . http://www.isc.hbs.edu/pdf/Finnish_Health_Care_System SITRA2009.pdf Accessed 2 October $201 \overline{3}$

7. World Health Organisation (WHO) (2012): Home care across Europe 2012, current structure and future challenges. http://www.euro.who. int/_data/assets/pdf_file/0008/181799/e96757.pdf Assessed 13 October 2013

8. Hammar T, Perälä ML, Rissanen P (2009) Clients' and workers' perceptions on clients' functional ability and need for help: home care in municipalities. Scand J Caring Sci 23:21-32

9. National Institute for Health and Welfare (2012). Statistical yearbook on social welfare and health care 2012. http://urn.fi/URN:ISBN:978952-245-784-4 Accessed 2 October 2013

10. Sørbye LW, Hamran T, Henriksen N, Nordberg A (2010) Home care patients in four Nordic capitals - predictors of nursing home admission during one-year followup. J Multidiscip Healthc 3:11-18

11. Dimitrow M, Airaksinen M, Kivelä S-L, Lyles A, Leikola SN (2011) Comparison of prescribing criteria to evaluate the appropriateness of drug treatment in individuals aged 65 and older: a systematic review. J Am Geriatr Soc 59:1521-1530

12. The American Geriatrics Society (2012) Beers Criteria Update Expert Panel (2012) American Geriatrics Society updated Beers Criteria for potentially inappropriate medication use in older adults. J Am Geriatr Soc 60:616-631

13. Koecheler JA, Abramowitz PW, Swim SE, Daniels CE (1989) Indicators for the selection of ambulatory patients who warrant pharmacist monitoring. Am J Hosp Pharm 46:729-732 
14. Barenholtz Levy H (2003) Self-administered medication-risk questionnaire in an elderly population. Ann Pharmacother 37:982-987

15. Fuller D, Watson R (2005) Validating a self-medication risk assessment instrument. Clin Eff Nurs 9:78-83

16. Johnson M, Griffiths R, Piper M, Langdon R (2005) Risk factors for an untoward medication event among elders in community-based nursing caseloads in Australia. Public Health Nurs 22:36-44

17. Langford BJ, Jorgenson D, Kwan D, Papoushek C (2006) Implementation of a self-administered questionnaire to identify patients at risk for medication-related problems in a family health center. Pharmacotherapy 26:260-268

18. Gordon K, Smith F, Dhillon S (2007) Effective chronic disease management: patients' perspectives on medication-related problems. Patient Educ Couns 65:407-415

19. Pit SW, Byles JE, Cockburn J (2008) Prevalence of self-reported risk factors for medication misadventure among older people in general practice. J Eval Clin Pract 14:203-208

20. Gusdal AK, Beckman C, Wahlström R, Törnkvist L (2011) District nurses' use for an assessment tool in their daily work with elderly patients' medication management. Scand J Public Health 39:354360

21. Rovers J (2003) Hagel H (2012) Self-assessment tool for screening patients at risk for drug therapy problems. J Am Pharm Assoc 52: 646-652

22. Finnish National Board of Education (2011) Vocational Qualification In Social and Health Care, Practical Nurse 2010 , Regulation 17/011/ 2010 Publications 2011:21 http://www.oph.fi/download/140436 vocational_qualification_in_social_and_healthcare_2010.pdf Accessed $1 \overline{3}$ October 2013

23. Dalkey N, Helmer O (1962) An experimental application of the Delphi method to the use of experts. The RAND corporation, Santa Monica, California 1962, Available at: http://www.rand.org/content/ dam/rand/pubs/research_memoranda/2009/RM727.1.pdf. Accessed 23 January 2014

24. Mother D, Liberati A, Tetzlaff J et al for the PRISMA Group (2009) Preferred reporting items for systematic reviews and meta-analyses: the PRISMA statement. PLoS Med 2009;6;e1000097 http://www. plosmedicine.org/article/info\%3Adoi\%2F10.1371\%2Fjournal. pmed.1000097 Accessed 23 March 2013

25. Campbell SM, Cantrill JA (2001) Consensus methods in prescribing research. J Clin Pharm Ther 26:5-14

26. ${ }^{\circ}$ Metodix, Futunet, Internetix (2010) eDelfoi.fi 3.0 http://www. edelphi.fi/en/. Accessed 26 February 2011

27. Leikola SN, Tuomainen L, Ovaskainen H, Peura S, Sevòn-Vilkman N, Tanskanen P, Airaksinen MS (2009) Continuing education course to attain collaborative comprehensive medication review competencies. Am J Pharm Educ 73:108
28. Sinnemäki J, Sihvo S, Isojärvi J, Blom M, Airaksinen M, Mäntylä A (2013) Automated dose dispensing service for primary healthcare patients: a systematic review. Syst Rev. doi:10. 1186/2046-4053-2-1

29. Lampela P, Hartikainen S, Sulkava R, Huupponen R (2007) Adverse drug effects in elderly people - a disparity between clinical examination and adverse effects self-reported by the patient. Eur J Clin Pharmacol 63:509-515

30. Cantrill JA, Sibbald B, Buetow S (1998) Indicators of the appropriateness of long-term prescribing in general practice in the United Kingdom: consensus development, face and content validity, feasibility, and reliability. Qual Health Care 7:130-135

31. Morris CJ, Cantrill JA (2003) Preventing drug-related morbidity - the development of quality indicators. J Clin Pharm Ther 28:295-305

32. Rognstad S, Brekke M, Fetveit A, Spigset O, Wyller TB, Straad J (2009) The Norwegian General Practice (NORGEP) criteria for assessing potentially inappropriate prescriptions to elderly patients. A modified Delphi study. Scand J Prim Health Care 27:153-159

33. Fick DM, Cooper JW, Wade WE, Waller JL, Maclean JR, Beers MH (2003) Updating the Beers criteria for potentially inappropriate medication use in older adults: results of US consensus panel of experts. Arch Intern Med 163:2716-2724

34. Laroche ML, Charmes JP, Merle L (2007) Potentially inappropriate medications in the elderly: a French consensus panel list. Eur J Clin Pharmacol 63:725-731

35. Gallagher P, Ryan C, Byrne S, Kennedy J, O'Mahony D (2008) STOPP (Screening Tool of Older Person's Prescriptions) and START (Screening Tool to Alert doctors to Right Treatment). Consensus validation. Int J Clin Pharm Ther 46:72-83

36. Budnitz DS, Lovegrove MC, Shehab N, Richards CL (2011) Emergency hospitalizations for adverse events in older Americans. N Engl J Med 365:2002-2012

37. Akins RB, Tolson H, Cole BR (2005) Stability of response characteristics of a Delphi panel: application of bootstrap data expansion. BMC Med Res Methodol 1(5):37

38. Beers MH (1997) Explicit criteria for determining potentially inappropriate medication use by the elderly: an update. Arch Intern Med 157:1531-1536

39. Stefanacci RG, Cavallaro E, Beers MH, Fick DM (2009) Developing explicit positive beers criteria for preferred central nervous system medications in older adults. Consult Pharm 24:601-610

40. Dimitrow M, Leikola S, Kivelä SL, Airaksinen M, Mykkänen S, Puustinen J (2013) Inappropriate medication use among the aged. Review of the criteria. Duodecim. 129:1159-1566 Review. Finnish, Abstract in English 